\section{Morphological Variation of Five Cut Chrysanthemum Cultivars Induced by 5-Azacytidine Treatment}

\author{
Dongru Kang, Silan Dai ${ }^{1}$, Kang Gao, Fan Zhang, and Hong Luo \\ Beijing Key Laboratory of Ornamental Plants Germplasm Innovation \\ \& Molecular Breeding, National Engineering Research Center for \\ Floriculture, Beijing Laboratory of Urban and Rural Ecological \\ Environment, Key Laboratory of Genetics and Breeding in Forest Trees \\ and Ornamental Plants of Ministry of Education, School of Landscape \\ Architecture, Beijing Forestry University, 35 East Tsinghua Road, Beijing \\ 100083, China
}

Additional index words. cut chrysanthemum, flowering time, 5-azacytidine, DNA methylation, methyltransferase inhibitor, flower induction

\begin{abstract}
In this study, five cultivars of cut chrysanthemum Chrysanthemum $\times$ morifolium Ramat., 'Jinba', 'Yuuka', 'Fenguiren', 'Xueshen', and 'Huangjin' were used to explore the functions of 5-azacytidine (5-azaC) on chrysanthemum growth and flower development. The results showed that 5-azaC had different effects on the growth of the five cultivars during in vitro culture. The final statistics showed that low concentrations promoted plant growth, whereas high concentrations inhibited growth; however, each cultivar had different growth curves, demonstrating that 5-azaC had no consistent inhibitory actions on growth. On the basis of the squaring time and flowering time statistics, we found that 5-azaC had a certain effect on the flowering time of all cut chrysanthemums, and all of these cultivars showed extremely early strains. Summer chrysanthemum ('Yuuka', 'Fenguiren', 'Xueshen', and 'Huangjin') treatments led to both early and delayed flowering. When the statistics were analyzed for different individuals, we found that the treatments shortened the squaring time in early-flowering plants. In 'Jinba', an autumn chrysanthemum, the treatment helped broken juvenile limitations and allowed plants to undergo photoperiod induction in the early stage. Additionally, we also determined the flower diameter differences in these treatments; ray florets from 'Yuuka' and 'Huangjin' trended to show tubular florets, and the location of tubular and ray florets were changed in 'Xueshen' capitulum. In conclusion, on the basis of flowering time in five early varieties of cut chrysanthemum, we propose that 5-azaC may regulate the methylation level of genes that control flower induction and flower development. These results provide phenotypic data and material for exploring the function of DNA methylation in regulating flowering.
\end{abstract}

The plant flowering process is achieved by integrating multiple internal and external flowering signals by regulating gene expression patterns (Bratzel and Turck, 2015). Multiple genes control the regulation of plant flowering (Blümel et al., 2014). The flowering process involves not only the upstream and downstream interactions of multiple genes but also a variety of apparent regulatory mechanisms (Deng and Cao, 2007). Epigenetic mechanisms play a key regulatory role in angiosperm flowering signaling pathways (Zografou and Turck, 2013), such as DNA methylation (Gao, 2012; Song, 2013; Xiao, 2010), RNAbased mechanisms (Sun et al., 2015; Teotia and Tang, 2015), and histone modification

Received for publication 12 Mar. 2019. Accepted for publication 2 Apr. 2019

This research was funded by National Natural Science Foundation of China, grant number 31530064

1Corresponding author. E-mail: silandai@sina.com. er than the probability of methylation, which ultimately leads to a significant decrease in methylation levels (Yuan et al., 2012).

Accordingly, many scholars have used DNA methyltransferase inhibitors to treat plants (Fieldes and Amyot, 1999; Kondo et al., 2006; Kumpatla and Hall, 1998; Li et al., 2015; Tyunin et al., 2012; Wang et al., 2009), expecting to obtain differential phenotypes for further analysis of possible epigenetic mechanisms. As early as 1993, Burn and colleagues used 5-azaC treatments on Arabidopsis thaliana and found that 5-azaC could replace the effect of low temperature and promote early flowering in Arabidopsis plants requiring vernalization. However, vernalization-insensitive Arabidopsis did not respond to 5-azaC, indicating that decreasing methylation levels can promote plant flowering instead of low temperature (Burn et al., 1993); this effect was also demonstrated by similar methods in species (Kondo et al., 2006, 2007). In chrysanthemum, 5-azaC treatment was also found to cause changes in the flowering time (Li et al., 2017; Wang et al., 2009; Zhu, 2014). In spinach, 5-azaC not only affected the flowering time but also had specific effects on spinach sexual performance, which significantly increased the percentage of monoecious individuals ( $\mathrm{Li}$ et al., 2015). In wheat, low concentrations of 5 -azaC increase the tillering ability of wheat and also affect the heading and flowering period, 1000-grain weight, and other indicators. High concentrations of 5-azaC inhibit the growth of wheat roots and seedlings (Chen and Wang, 2011). Additionally, 5-azaC treatment promotes Stellaria longipes stem elongation (Tatra et al., 2000), suggesting that DNA methylation is involved in the regulation of plant shade responses. 5-azaC binding to phytohormone 2.4-D under tissue culture conditions also increases the development of somatic embryos in Acca sellowiana (Fraga et al., 2012).

Additionally, previous studies have found that changes in the methylation levels of key genes can also cause phenotypic variation. For example, the blue fluorescence of Arabidopsis thaliana mutants is due to the methylation horizontal variation of the PAI (phosphoribosylanthranilate isomerase) gene, rather than mutations at this locus (Martienssen, 1996; $\mathrm{He}$ and $\mathrm{Li}, 2001$ ), in the peloria flower of Linaria vulgaris is due to the hypermethylated of the CYC gene (Cubas et al., 1999). Among the epigenetic mutants associated with Arabidopsis flowers, the SUPERMAN gene shows a variation in DNA methylation levels (Jacobsen and Meyerowitz, 1997). These examples show that the phenotype caused by the variation in the methylation level of the coding gene DNA sequence is consistent with the variation type of the mutation in the gene locus.

Studies on plant DNA methylation vs. flowering have focused on DNA methyltransferase inhibitors to treat plants and to observe correlations between phenotypic traits and methylation levels. Moreover, 
in-depth mechanism studies have been conducted on the apparent mutants that have been identified. Although DNA methylation has been found to be associated with many biological processes, its specific mechanism of action has not been thoroughly elucidated to date.

Chrysanthemum $\times$ morifolium Ramat. is the most popular market-oriented chrysanthemum strain, and its flowering time regulation is the most economical breeding direction (Zhang and Dai, 2013). At present, the flowering time regulation of chrysanthemum in industrial production mainly depends on shading and fill light. If a cut flower chrysanthemum strain with a short photoperiod induction in days could be obtained, the production costs and the damage from pests and diseases caused by shade can be greatly reduced. In this study, we examined the main varieties used for the production of cut chrysanthemums, 'Yuuka', 'Huangjin', and 'Jinba', and two new varieties, 'Fenguiren' and 'Xueshen', with independent intellectual property rights cultivated by our research group under tissue culture conditions using 5-azaC treatment. We sought to obtain a cut chrysanthemum strain with a modified flowering stage. Phenotypic analysis of the treated progeny will be performed to explore the effects of DNA methyltransferase inhibitors on the growth and development of cut chrysanthemums. The research materials were provided for the regulation of chrysanthemum flowering and the regulation of flower development.

\section{Materials and Methods}

\section{Plant materials}

The materials used in this study include the main varieties used for the production of cut chrysanthemums, including 'Yuuka', 'Huangjin', and 'Jinba', and two new varieties, 'Fenguiren' and 'Xueshen', with independent intellectual property rights cultivated by our group. The five cut chrysanthemum varieties are maintained in our laboratory. The stem segments from the individuals were selected, and different concentrations of 5-azaC were used for treatment under tissue culture conditions.

5-azaC (CAS:320-67-2) was purchased from Takara Co., Ltd. Treatment with 5-azaC was performed on stem segments from single-bud tissue culture plants $(5$-azaC for filter sterilization). The following concentrations of 5-azaC were added to the Murashige and Skoog (MS) medium (MS $+30 \mathrm{~g} \cdot \mathrm{L}^{-1}$ sucrose $+6 \mathrm{~g} \cdot \mathrm{L}$ Agar, $\mathrm{pH}$ 5.8): 10, 20, 50, $100,150,200$, and $400 \mu \mathrm{mol} \cdot \mathrm{L}^{-1}$. The method for treating the cut chrysanthemum using 5azaC is shown in Table 1. For each of the cut chrysanthemum varieties, treatment included three to five replicates with six inoculated stem segments per bottle with $50 \mathrm{~mL}$ MS medium (bottle capacity $240 \mathrm{~mL}$ ). After $50 \mathrm{~d}$ of treatment, the stem segments were moved to an artificial climate chamber (12,000 Lux, $25 \pm 2{ }^{\circ} \mathrm{C}, 12 \mathrm{~h} \mathrm{light} / 12 \mathrm{~h}$ dark).

Table 1. Method for treating cut chrysanthemums with 5-azaC.

\begin{tabular}{llllllllcc}
\hline & \multicolumn{10}{c}{ Code } \\
\cline { 2 - 10 } 5-azaC treatment & 0 & 1 & 2 & 3 & 4 & 5 & 6 & 7 & 8 \\
\hline Concentration $\mu \mathrm{mol} \cdot \mathrm{L}^{-1}$ & 0 & 10 & 20 & 50 & 100 & 150 & 200 & 400 & 800 \\
Xueshen & $\sqrt{ }$ & $\sqrt{ }$ & $\sqrt{ }$ & $\sqrt{ }$ & $\sqrt{ }$ & $\sqrt{ }$ & $\sqrt{ }$ & $\sqrt{ }$ & $\sqrt{ }$ \\
Fenguiren & $\sqrt{ }$ & $\sqrt{ }$ & $\sqrt{ }$ & $\sqrt{ }$ & $\sqrt{ }$ & $\sqrt{ }$ & $\sqrt{ }$ & $\sqrt{ }$ & - \\
Huangjin & $\sqrt{ }$ & $\sqrt{ }$ & $\sqrt{ }$ & $\sqrt{ }$ & $\sqrt{ }$ & $\sqrt{ }$ & $\sqrt{ }$ & $\sqrt{ }$ & - \\
Yuuka & $\sqrt{ }$ & $\sqrt{ }$ & $\sqrt{ }$ & $\sqrt{ }$ & $\sqrt{ }$ & $\sqrt{ }$ & $\sqrt{ }$ & $\sqrt{ }$ & - \\
Jinba & $\sqrt{ }$ & $\sqrt{ }$ & $\sqrt{ }$ & $\sqrt{ }$ & $\sqrt{ }$ & $\sqrt{ }$ & $\sqrt{ }$ & $\sqrt{ }$ & - \\
\hline
\end{tabular}

\section{Growth data statistics and analysis}

Growth data before transplanting. The growth indicators for the cut chrysanthemum seedlings to be transplanted included the following statistical indicators: plant height, number of leaves, root length (longest root hair), and number of root hairs.

The growth data during transplanting were performed on the following strains: 'Jinba' (163), 'Xueshen' (126), 'Fenguiren' (190), 'Yuuka' (128), and 'Huangjin' (117), with 724 strains in total.

Observation of squaring and flowering time. All cut chrysanthemums were moved to an artificial climate chamber for short daylight $\left(12,000\right.$ Lux $25 \pm 2{ }^{\circ} \mathrm{C}, 12 \mathrm{~h}$ light/ $12 \mathrm{~h}$ dark) treatment, and the squaring and flowering of the plants were observed. The statistical methods examined the squaring time, which encompassed the time from the day of transfer to short daylight to the visible flower bud (diameter $1 \mathrm{~mm}$ ) and the flowering time, the time from the day of transfer to short daylight to the initial bloom of the flower. The squaring time and flowering time of the first flower from each plant in all treatments were counted, after which data analysis was performed.

Observation of other flower traits. The flower diameter, number of flowers, and number of florets (each capitulum) from the flowering plants were counted, and the height of the plants, the number of leaves, and the crown width of the flowering plants were also measured.

Statistics and data analysis. All data were calculated using SPSS24 for the mean and standard error, and Excel 2016 was used to draw the histograms and dot plots.

\section{Results}

\section{Effects of 5-azaC treatment on the} vegetative growth of different varieties of cut chrysanthemum

The growth indicators measured in all the cut chrysanthemum showed no consistency with the 5-azaC concentration change (Fig. 1). Different plant growth was observed under treatment with different concentrations of 5-azaC (inhibition or promotion).

In summary, the sensitivity and trends of different cut chrysanthemums to 5-azaC are not the same because the growth indicators are not consistent. Considering the uncertainty of the role of 5-azaC on the chrysanthemum genome, we focused on further analysis and studied the observable flowering and flower phenotypic traits in the mutant library.

\section{Effects of 5-azaC treatment on the flowering rate of different varieties of cut chrysanthemum}

Statistical analysis of the flowering rate showed that the effect of 5 -azaC on the induction of flowering in the different cut chrysanthemums was not the same. For 'Xueshen', the flowering rates in several treatments were lower than in the control, but all were higher than $92 \%$, and the difference was not significant (Fig. 2A) The treatments except for treatment 6 and 7 in the 'Yuuka' group were lower than in the control (Fig. 2B). The flowering rate of 'Yuuka' was similar to that of 'Huangjin', although several treatments were lower than the control, and lowest flowering rate was in treatment 3 . The treatments except for treatment 5, 6, and 7 in the 'Huangjin' group were lower than in the control (Fig. 2C). Thus, it can be observed that 5-azaC has varietyspecific effects on the flowering of cut chrysanthemums.

In 'Jinba', only five plants in treatment 6 (20 individuals) underwent flowering, with a flowering rate of $25 \%$, and none of the other treatments flowered (Fig. 2D). 'Jinba' is a strict autumn chrysanthemum variety that has the longest childhood or photoperiod-induced days, which potentially indicates why the control and other treatments did not undergo flowering. Several plants in treatment 6 could break through the childhood restriction and form a flower after receiving a certain number of photoperiod induction days. We speculate that the gene affected by 5 -azaC plays a key role in the flower induction process, which can promote the younger 'Jinba' to flowering. Thus, 'Jinba' could overcome the childhood restriction and then accept the photoperiod induction, leading to an early flowering phenomenon.

\section{Effects of 5-azaC treatment on the flowering time of different varieties of cut chrysanthemum}

Among all the treatments for 'Xueshen', only treatment 7 showed a lower flowering time than the control (Fig. 3A). The flowering time for 'Xueshen' ranged from 79 to $188 \mathrm{~d}$ $\left(\bar{X}_{0}=108 \mathrm{~d} ; \bar{X}_{0}\right.$ represents the average flowering time of the control). For 'Yuuka', only the flowering time of treatment 5 was shortened; the other treatments delayed the flowering time (Fig. 3B), leading to a flowering time range from 57 to $179 \mathrm{~d}\left(\overline{\mathrm{X}}_{0}=102 \mathrm{~d}\right)$ for 'Yuuka'. For 'Huangjin', treatments 4 and 6 more shortened the flowering time compared with the control (Fig. 3C). The radiant time range for 'Huangjin' is 106 to $196 \mathrm{~d}\left(\overline{\mathrm{X}}_{0}=157 \mathrm{~d}\right)$. Treatments 3 

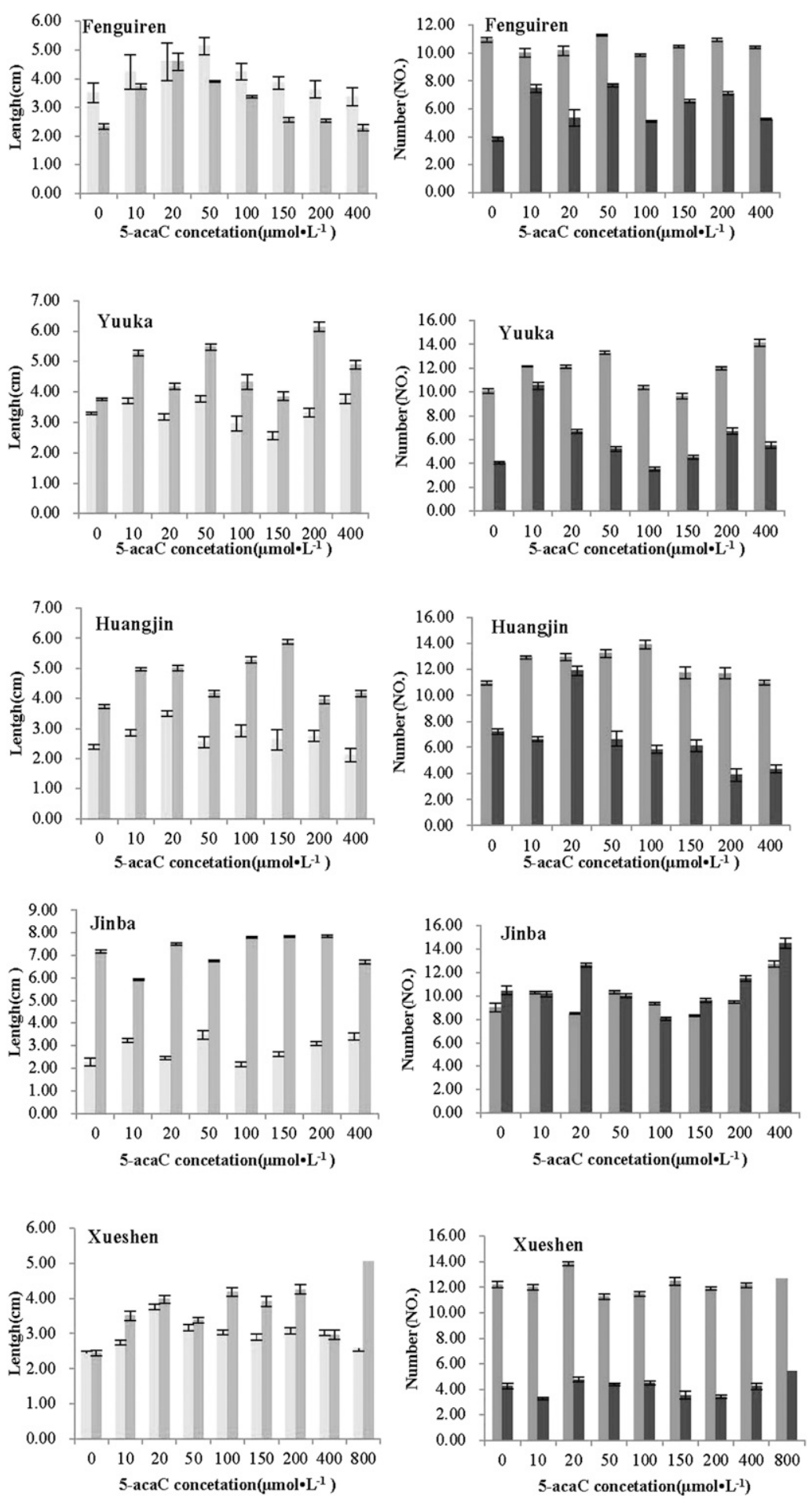

$$
\begin{array}{lll}
\text { Plant Hight } & \text { Root Length } & \text { Lear Number } \\
\text { Root Number }
\end{array}
$$

Fig. 1. Effect of different concentrations of 5-azacytidine (5-zazC) on the growth of cut chrysanthemums.

and 5 had the shortest flowering time in 'Fenguiren' (Fig. 3D); all the other flowering periods were delayed, leading to a flowering time range from 79 to $173 \mathrm{~d}\left(\overline{\mathrm{X}}_{0}=152 \mathrm{~d}\right)$ in 'Fenguiren'. In 'Jinba', only five plants in treatment 6 underwent flowering, displaying a mean flowering time of $103 \mathrm{~d}$; thus, the flowering time for this variety ranged from 93 to $111 \mathrm{~d}$. Some flowering time data for
'Fenguiren' and 'Yuuka' were not collected due to natural factors (Fig. 3).

Early flowering lines were obtained in all cut chrysanthemums, but at the same time, some individuals also showed delayed flowering times. There were no clear trends, and the flowering period distribution was wide. Therefore, the mean values for each treatment can only characterize the specific pop- ulations. The analysis should be considered for differences in the flowering time among individual plants.

\section{Effects of 5-azaC treatment on flowering time in different individual 'Xueshen' plants}

We wanted to know which part of flower development under 5-azaC treatment is shortened in the early flowering line, leading to early flowering. Therefore, further analysis was performed on the data from the squaring time and flowering time in 'Xueshen'.

Because flower development is difficult to capture, we used the visible squaring time to visualize the length of flower induction time and the time from the bud to the opening of the flower to characterize the flower development time. In fact, flowering induction has been completed at the time of the squaring and includes some floral development time.

A histogram is drawn for the flowering, squaring, and flower development times in 'Xueshen' (Fig. 4). The flowering times and the squaring times for all the 'Xueshen' treatments are consistent (Fig. 4A and B). In the flower development time analysis, we found no significant differences in the flower development times between the treatments and the control group (Fig. 4C). The decisive factor in the mechanism of early flowering in 'Xueshen' is the length of the flowering induction time.

Given the preceding definition, a comparative analysis of the photoperiod induction days and flower development time between individual individuals in the mutant library was performed. First, the squaring, flowering, and flower development times of all individuals in the 'Xueshen' population underwent statistical analysis. This analysis was performed on a per-plant basis, meaning that all the individuals were sorted and plotted according to their squaring time.

According to the preceding data, the average flowering period in the mutant library (either the squaring time or the flowering time) can be used to evaluate the flowering period of the mutant progeny to some extent. However, there are differences among individuals in the same treatment. For example, for 'Xueshen' treatment 8 (Fig. 5A), the flowering time distribution was 72 to $113 \mathrm{~d}$, and all of the flowering period data are scattered. Four of these individuals' flowering times were more significantly advanced, ranging between 70 and $80 \mathrm{~d}$ (Fig. 5A, red circle), whereas the flowering times of 10 individuals were between 90 and $100 \mathrm{~d}$; moreover, the other 10 individuals had flowering times at $\approx 110 \mathrm{~d}$ (Fig. 5A, blue circle). Thus, even in progeny treated at the same concentration, the flowering time distribution is relatively scattered. Therefore, the flowering period of the evaluation mutant library should be measured by the flowering time of a single individual. That is, the sensitivity of a photoperiod response between different plants in the same concentration treatment is not completely consistent. In view of the randomness of 5-azaC inhibition 
of gene methylation, different sites may occur between different plants. Because the DNA methylation levels may reduce as the 5azaC treatment, the flowering period should be analyzed from the perspective of the flowering times of different plants.
In Fig. 5B, the squaring time is sorted from early to late. For the early flowering line from 'Xueshen', 5-azaC shortened its photoperiod induction days, meaning that it required fewer photoperiod induction days to its squaring time, although its flower devel-

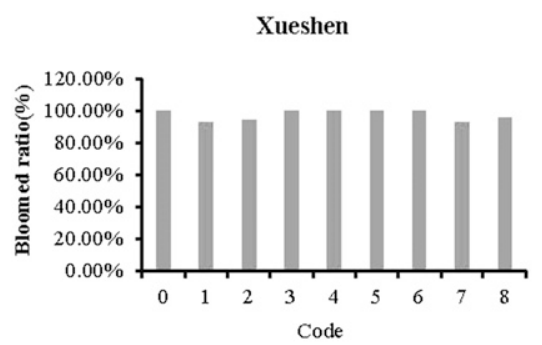

(A)

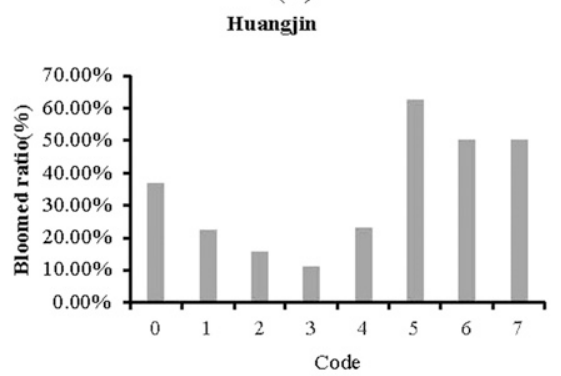

(C)

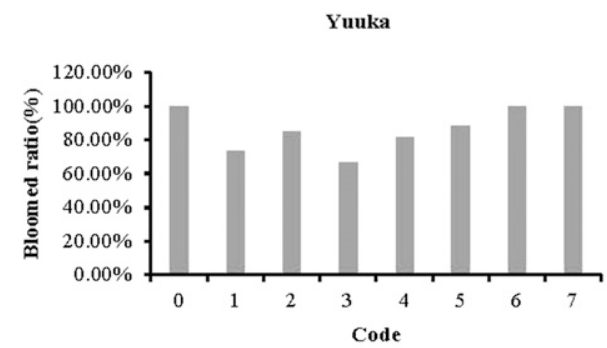

(B)

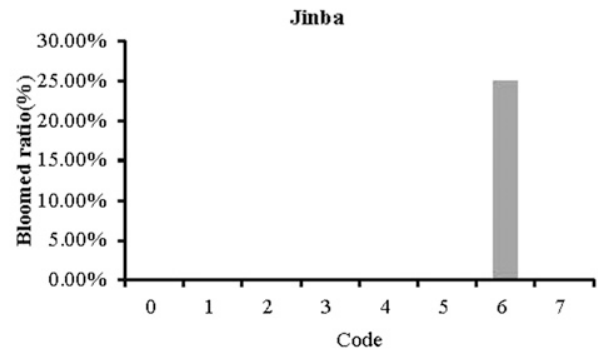

(D)

Fig. 2. Statistical analysis on the flowering rate of different cut chrysanthemums.

opment still needed to be stable (Fig. 5B). The analysis of the flowering time of different plants compared with the mean flowering time among treatments can better capture the differences in the developmental stages of the early and late flowering lines.

Correlation analysis between the growth and flowering stage of cut chrysanthemums

The squaring and flowering times of the treated 'Huangjin' progeny lines (Fig. 6A) show that 'Huangjin' individuals have a relatively stable flower development time. The flower development and squaring times showed differences, with the earliest flowering line having fewer flowering photoperiod days, although its flower development period was prolonged. However, although the number of days for the photoperiod induction increased gradually, the flower development time did not show a significantly changed trend, indicating its stable value. Therefore, the effect of 5-azaC on the flowering time of 'Yuuka' can be observed, as the flower development time did not change significantly, whereas the squaring time changed significantly (Fig. 6B). Thus, 5-azaC treatment shortened or lengthened the photoperiod induction time in 'Xueshen', 'Huangjin', and 'Yuuka'.

For the very early flowering plants among the cut chrysanthemums treated with 5-azaC,

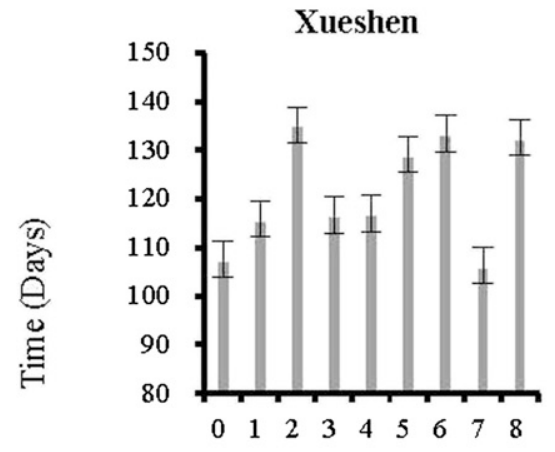

(A)

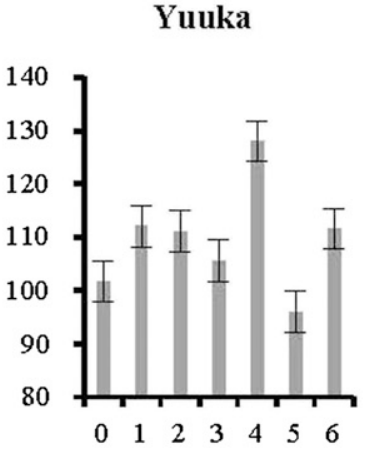

(B)
Huangjin

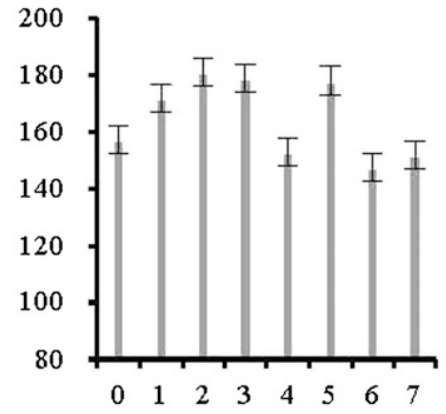

(C)
Fenguiren

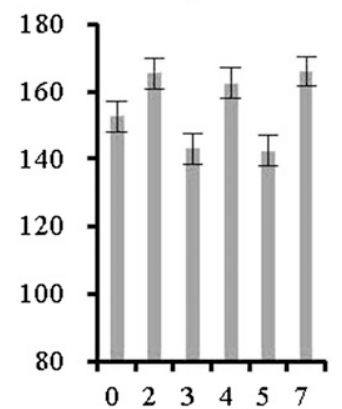

(D)

Fig. 3. Differences in flowering time due to different treatments.

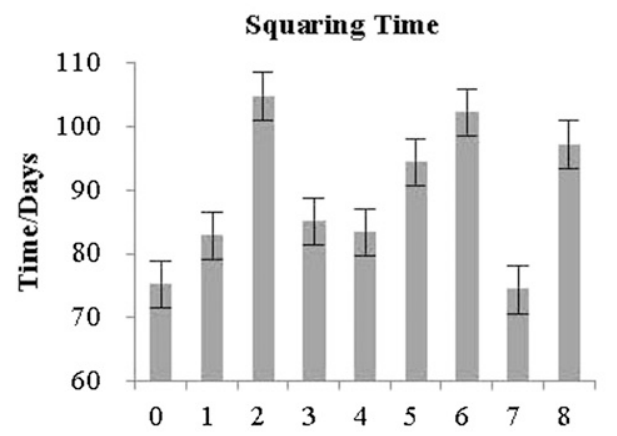

(A)

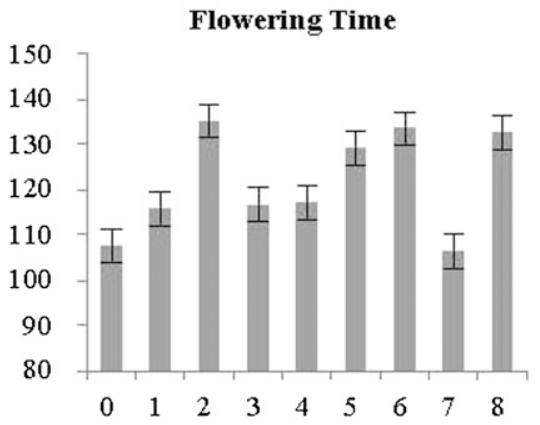

(B)
Floral Development

Time

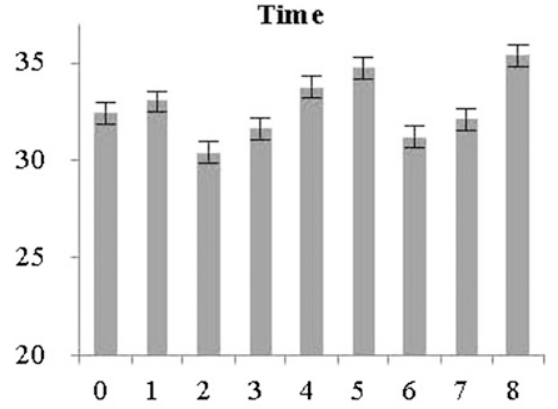

(C)

Fig. 4. Flowering, squaring, and floral development times in 'Xueshen'. 
the photoperiod response days were the shortest. Although some flower development periods were relatively long, the overall performance showed the earliest flowering stage. Therefore, we speculate that treatment with 5-azaC inhibits DNA methylation maintenance in genes associated with the flowering-induced regulatory network, resulting in a decrease in the methylation levels of some genes, which finally leads to specific early flowering in some cut chrysanthemum flower strains. On this basis, screening key genes regulated by DNA methylation in flowering regulatory networks is particularly important. Therefore, studies on the regulation mechanism of DNA methylation in photoperiod induction of chrysanthemum should be performed.

\section{Correlation analysis between the growth and flowering stage in cut chrysanthemum} leaves) of different cultivars in the cut chrysanthemum population was analyzed, and two trends were found. The squaring time of 'Fenguiren' and 'Huangjin' was positively correlated with plant height and leaf number (Fig. 7), whereas the squaring time of
The growth (plant height and number of

'Yuuka' and 'Xueshen' was negatively correlated with plant height and leaf number (Fig. 7).

We speculate that the summer chrysanthemum varieties ('Yuuka' and 'Xueshen') grow to a specific vegetative growth stage and then undergo flowering induction, in which its own nutrient conditions perform the dominant role in flowering induction. Thus, the length of its youth limits important factors in its light response. Summer chrysanthemum is able to maintain sufficient growth to maintain flowering even under short-day conditions.

Even if the summer and autumn chrysanthemum varieties ('Fenguiren' and 'Huangjin') meet the growth conditions induced by photoperiod, they still require a specific number of short days to undergo flowering induction. It is speculated that the main factor limiting the growth of summer-autumn chrysanthemums is the photoperiod induction day. Thus, the limiting factor for summer and autumn chrysanthemum varieties is their own nutritional conditions and photoperiod induction days. Additionally, we speculate that the photoperiod-inducing pathway (response to short day) and autonomous pathway coexist in the flowering induction

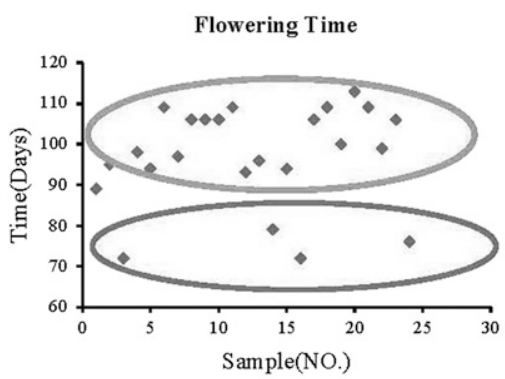

(A)

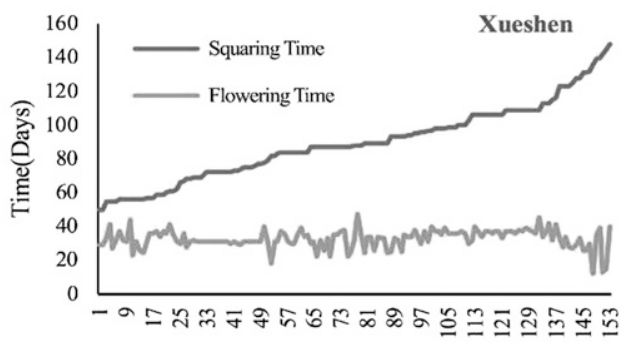

Sample(NO.)

(B)
Fig. 5. Squaring, flower development, and flowering time distributions for 'Xueshen'. (A) Top circle indicates early flowering times; the circle below indicates delayed flowering times. (B) The squaring time is sorted from early to late. pathway in summer and autumn chrysanthemum (responding to nutritional status).

\section{Effects of 5-azaC treatment on flower morphology in different varieties of cut chrysanthemum}

By analyzing the flower diameter and number of florets in the offspring of cut chrysanthemums, we found that different 'Yuuka' cut chrysanthemum plants treated with 5 -azaC showed significant differences in size (Fig. 8).

Therefore, the flower diameter and number of florets in all the cut chrysanthemum flowering plants were statistically analyzed (Table 2). The $\mathrm{CV}$ (coefficient of variation) for the flower diameter in 'Yuuka' was $29.85 \%$, whereas the $\mathrm{CV}$ for florets number was $30.26 \%$. The $\mathrm{CV}$ was the largest for this cut chrysanthemum, indicating that its flower traits are most the unstable under 5-azaC treatment. The $\mathrm{CV}$ for the diameter of the 'Huangjin' flower was $10.38 \%$, and the $\mathrm{CV}$ for the number of florets was $19.04 \%$. The variation coefficient of flower diameter in 'Fenguiren' was $16.77 \%$, and the $\mathrm{CV}$ for florets number is $29.21 \%$ (Table 2).

The $\mathrm{CV}$ for flower diameter in 'Xueshen' is $8.50 \%$, whereas for florets, it was $16.42 \%$. The $\mathrm{CV}$ in 'Xueshen' was lowest, making it our preferred variety. The stability of its flower shape is superior to the popular Japanese chrysanthemum variety 'Yuuka' and the Chinese chrysanthemum variety 'Huangjin' (Table 2).

Tubular flowers and lingual flower positioning errors occurred in the treated offspring from 'Xueshen'. For example, round of tubular flowers (Fig. 9A) will occur between lingual flowers, and lingual flowers will also occur in the tubular flowers (Fig. 9B). This phenomenon is common in the cultivation of chrysanthemum, even in the natural state (Fig. 9C). The positioning of the lingual flower and the tubular flower is abrupt. It can be seen that the location of lingual flowers and tubular flowers may be

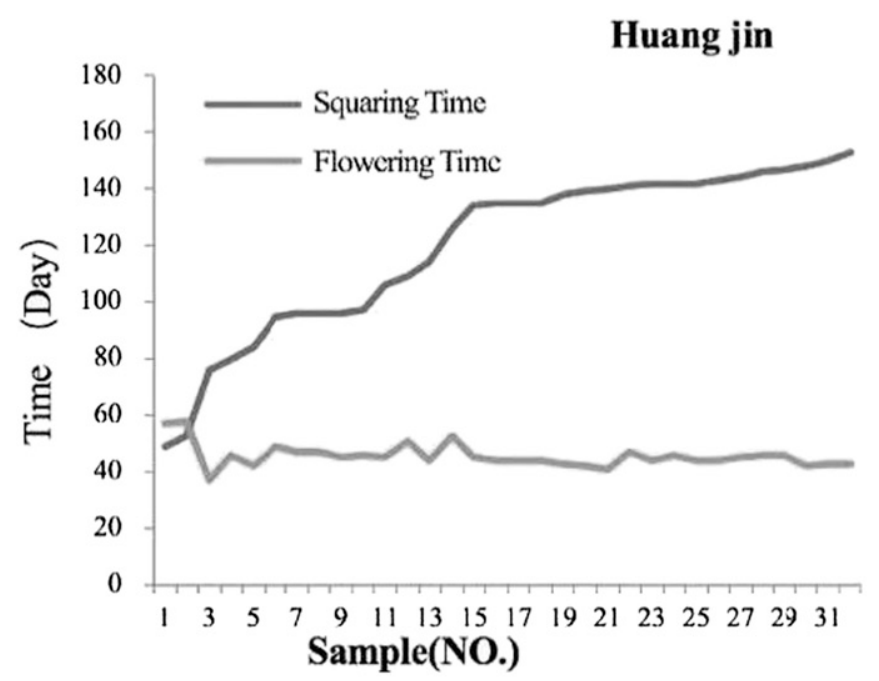

(A)

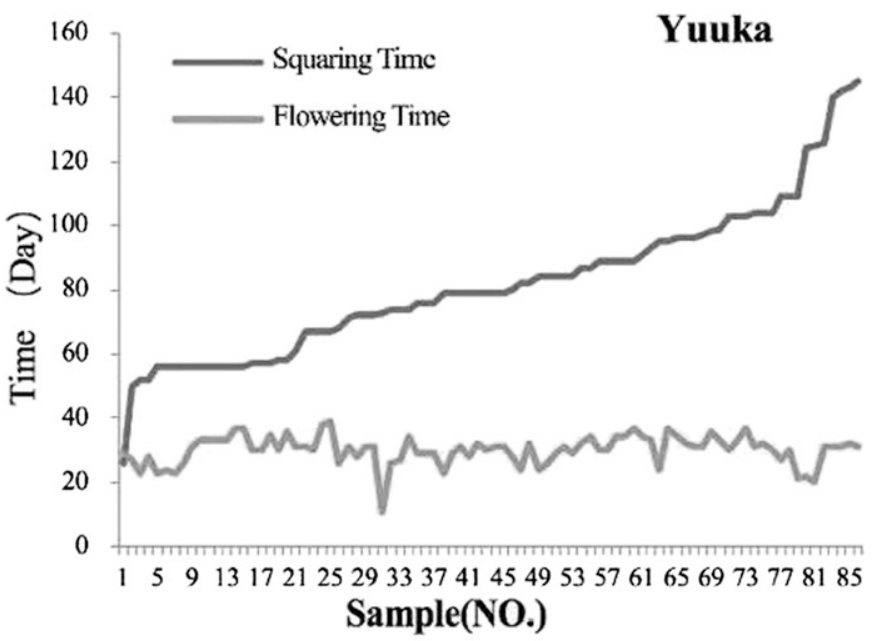

(B)

Fig. 6. Differences between the squaring and flower development times in 'Huangjin' and 'Yuuka'. 

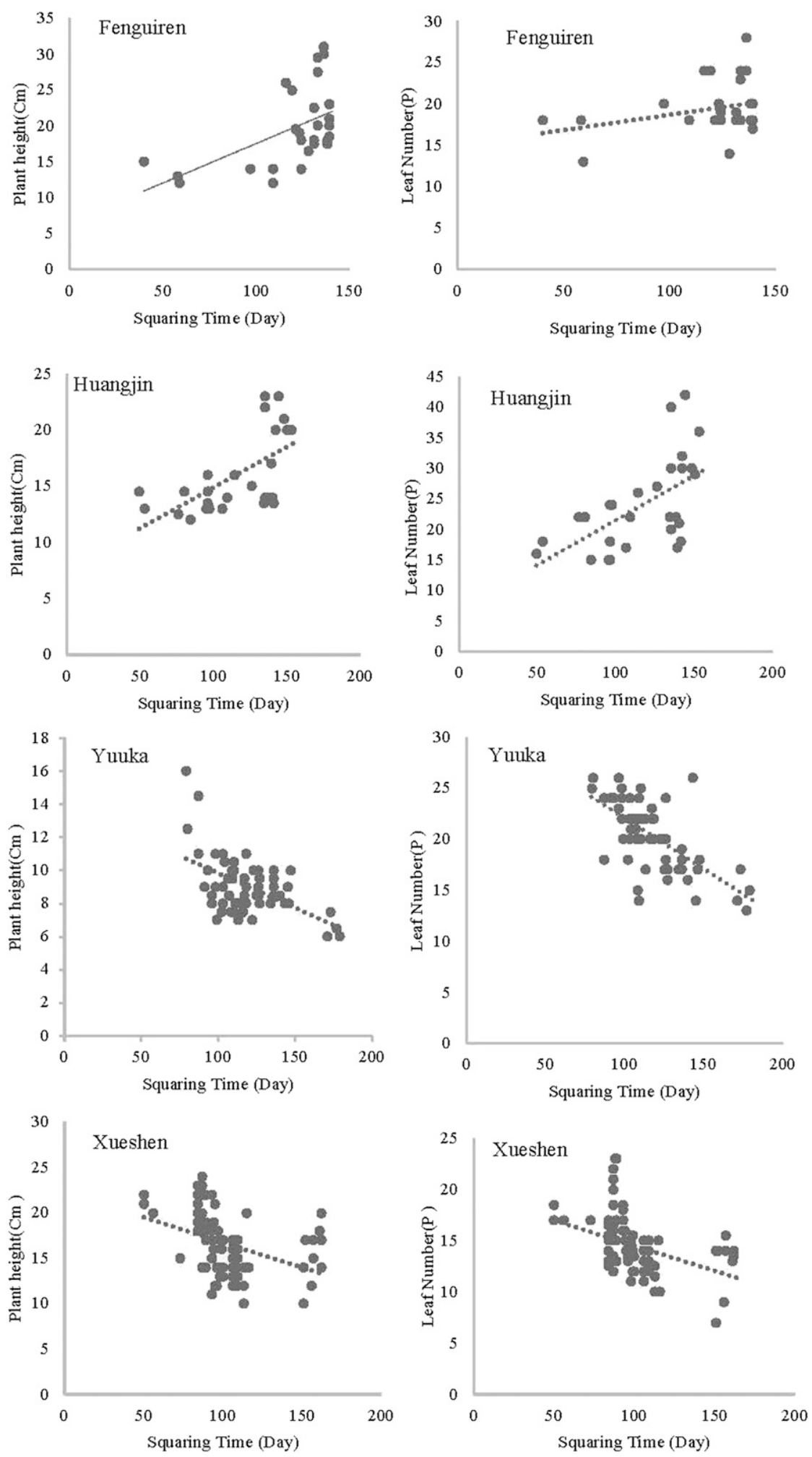

Fig. 7. Correlation analysis of squaring time and the growth of cut chrysanthemums.

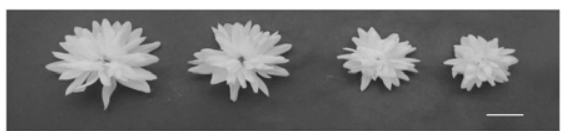

related to the localized expression of related genes that determine the type of florets. Research on the localization mechanism of lingual florets has yet to be studied in depth.

Cut chrysanthemum is the best-selling type of chrysanthemum in the modern cut flower market, and most of these, including 'Yuuka' (Fig. 9D), 'Huangjin' (Fig. 9E), and
Fig. 8. Differences in flower diameter in 'Yuuka'. Bars $=1 \mathrm{~cm}$.
'Jinba', have high-valve-type flat flaps. There is no development of the tubular flower. The preferred new variety, 'Xueshen', which our team has selected for breeding, the capitulum is green before opening (Fig. 9F). After opening, the capitulum is not exposed (Fig. 9G), and the flowers are white overall, like 'Yuuka'. The same is true for 'Fenguiren', in which the heart opens before the opening, the capitulum is pink, and they are not exposed after opening. The whole capitulum is pink after opening. Our proprietary varieties 'Xueshen' and 'Fenguiren' have tubular flowers that are not noticeable after the flowers are completely open, due to the high degree of doubling flower. 'Yuuka' ray floret morphology was normal; they are flat (Fig. 9H, left), and their base in some strains was closed (Fig. 9H, right). The flat florets have a tendency to be spoon florets, represented by the transition type between the flat and spoon florets in the inflorescence of cut chrysanthemum. The ray florets in 'Huangjin' also have this variation (Fig. 9-I).

The early flowering lines of 'Fenguiren' (FGR-5) showed fading and whitening after repeated passages (Fig. 10A). Additionally, the total bracts were born on the periphery of the ray flowers (Fig. 10B). In the early flowering lines (FGR-5), the bracts occurred between the ray flowers (Fig. 10D). In the capitulum, there are multiple rounds of bracts, and the ectopic bracts were smaller than the outermost bracts (Fig. 10C).

\section{Discussion}

\section{Randomness of 5-azaC processing on offspring}

5-azaC is a cytosine analog that inserts into DNA and binds to methyltransferase, thereby inhibiting the maintenance of methylation status. We aimed to determine whether 5-azaC acts as a specific reagent to improve plant flowering. Because the effect of the 5-azaC reagent on genes does not affect a specific gene, the affected genes may be different among strains. Some scholars have suggested that 5 -azaC can be used as a method to regulate flowering (Wang et al., 2009) and that silencing CmMET1 (DNA methyltransferase that maintains CG locus of DNA methylation) could decrease plant height, change the phenotype of chrysanthemum, and promote earlier flowering (Li et al., 2019), much like the morphological variation after 5-azaC treatment. We found that 5-azaC not only advances the flowering period in chrysanthemum but also delays the flowering period of some lines, showing that it is not ideal for affecting the flowering period. We should find ways to affect the flowering period specifically. In addition to 5-azaC's effect on flowering, it also promotes ectopic expression of the flat florets, which have a tendency to be spoon florets. It also causes changes in the color of $C$. lavandulifolium stems, and extremely short lines are also present (unpublished data). Because the DNA methylation mechanism involves various stages of plant development, 5-azaC 
causes not only flowering variation but also variations in other traits. Moreover, it causes not only early flowering but also a flowering delay. Therefore, 5-azaC cannot be used as a specific reagent to improve flowering, but it can be used as a methyltransferase inhibitor to establish the apparent mutant library and study DNA methylation in flowering regulation. The mechanism of action provides a breakthrough and important plant research material.

\section{Cut chrysanthemum 5-azac-treated early flowering line progeny with differ- ent reactions}

In the natural environment, the flowering time of cut chrysanthemums can be divided into summer, autumn, and winter chrysanthemums (Zhang and Dai, 2013). The difference in flowering season is due to the varying demands for photoperiod induction days among the varieties. In the offspring of cut chrysanthemums treated with 5-zazC, all cut chrysanthemums exhibited very early flower lines. The summer cut chrysanthemum early flower line showed a shortened flowering induction time, whereas the autumn chrysanthemum ('Jinba') overcame the childhood restriction and took advantage of early photoperiod induction. The summer and autumn chrysanthemums, which have differences in their photoreaction cycles, exhibit great differences in response to 5-azaC.

In daily production, our breeding aimed to shorten flowering and production cycles and reduce energy consumption. Changes of flowering time mainly depend on the induction of flower cycling and development time. The 5azaC-induced early flowering line achieves this purpose, but the genetic stability of its offspring requires further evaluation. How 5azaC regulates the specific regulation mechanisms of early flowering is also unclear.

\section{Studying the mechanism of early flowering lines}

In our study, the offspring from cut chrysanthemum treatment shortened the photope-

Table 2. Analysis of the variation coefficients in cut chrysanthemums

\begin{tabular}{lccccc}
\hline Variety & Sample number & Florets counts & Florets count cV & Flower diam $(\mathrm{cm})$ & Flower diam CV \\
\hline Yuuka & 76 & $77.81 \pm 0.33 \mathrm{~b}$ & $30.26 \%$ & $5.36 \pm 0.01 \mathrm{~b}$ & $29.85 \%$ \\
Xueshen & 29 & $135.00 \pm 0.76 \mathrm{a}$ & $16.42 \%$ & $3.85 \pm 0.01 \mathrm{c}$ & $8.50 \%$ \\
Huangjin & 26 & $131.42 \pm 0.96 \mathrm{a}$ & $19.04 \%$ & $6.10 \pm 0.02 \mathrm{a}$ & $10.38 \%$ \\
Fenguiren & 9 & $115.00 \pm 3.73 \mathrm{a}$ & $29.21 \%$ & $5.67 \pm 0.07 \mathrm{ab}$ & $11.67 \%$ \\
\hline
\end{tabular}

riod induction time or overcame childhood restriction, and achieved early photoperiod induction, leading to early flowering lines arising from all the cut chrysanthemum varieties. However, it is important to determine whether the early flower trait can be preserved through the subculture of tissue culture seedlings or vegetative propagation of foot buds or whether the early flower traits will decline after multiple passages; this requires further verification. It can be confirmed that 5-azaC treatment causes the flowering of the plant to be advanced or delayed because of methylation changes in flowering pathway genes or flower development genes under the action of the methyltransferase inhibitor; however, how the genes are regulated by DNA methylation remains unclear. Because of the uncertainty of the mechanism of action of 5 -azaC, it is possible that the genes regulated in the early flowering lines are not the same. Additionally, because the apparent regulation of plant genes exhibits spatiotemporal specificity, plants that undergo the vernalization pathway reprogram may have apparent imprints similar to the original apparent imprint state after low temperature induction (Crevillén et al., 2014). Moreover, the DNA methylation status of plants is dynamically changed. After flowering, some genes returned to their original DNA methylation state. However, after

$\mathrm{CV} \%=$ mean value/sD.
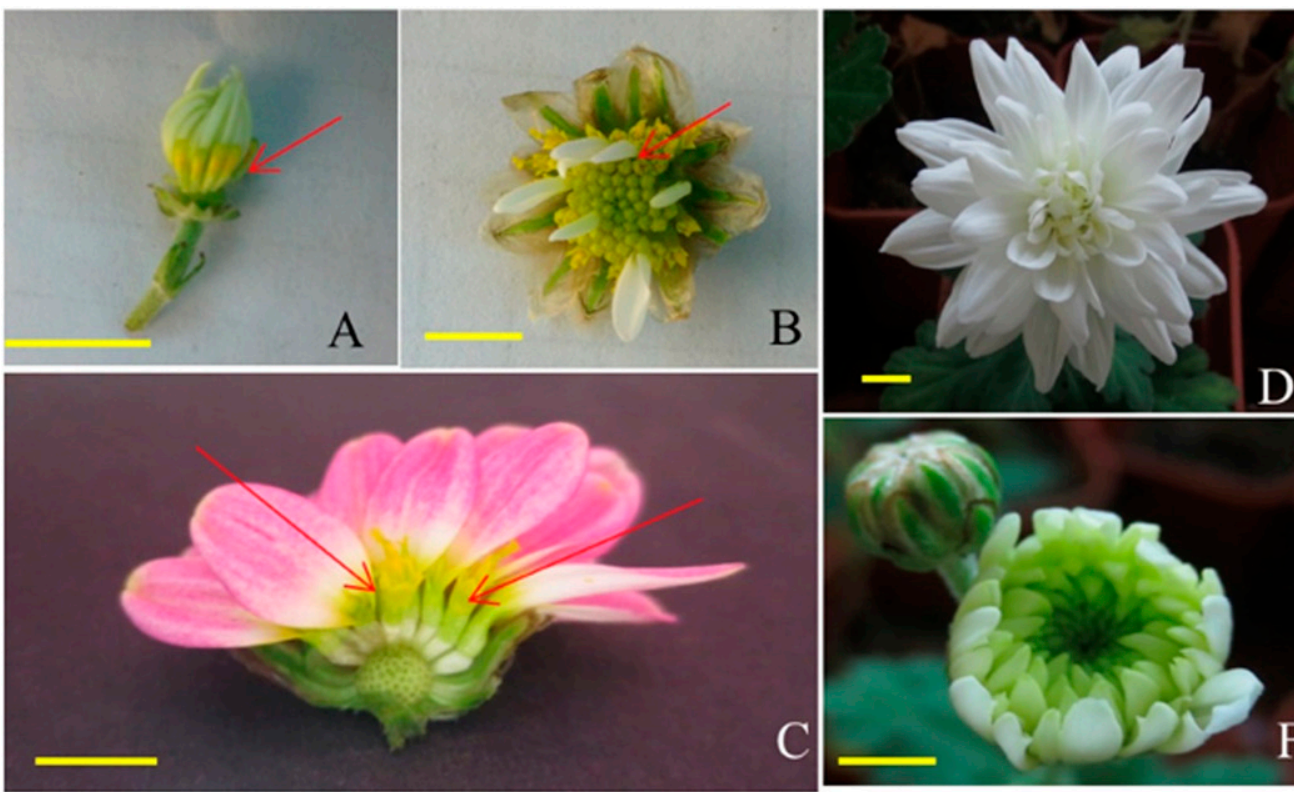

D
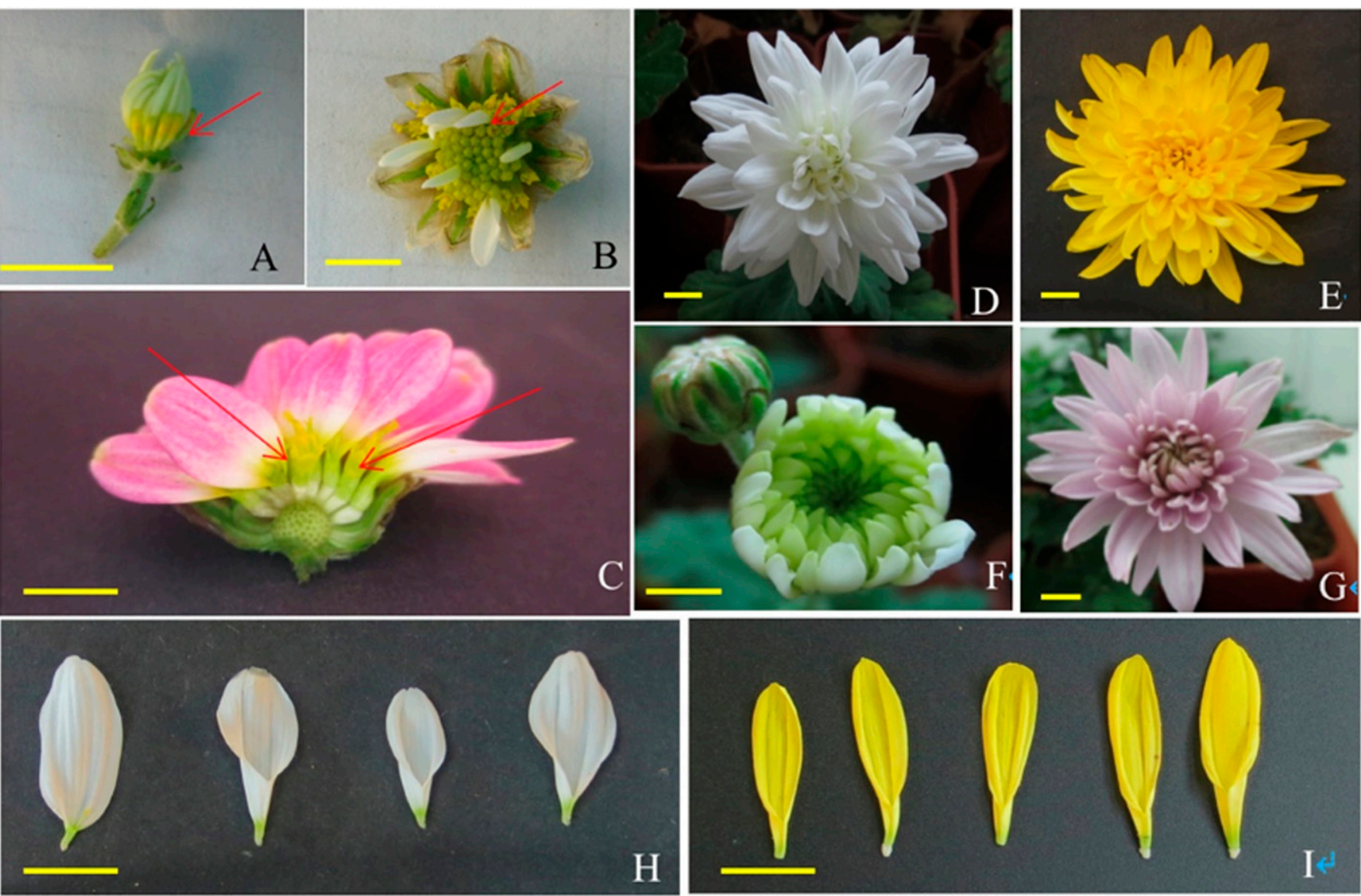

Fig. 9. Natural morphology and variation in cut chrysanthemum. (A-C) Floret location variation. (D-G) The natural morphology of cut chrysanthemum. (H and I) Variation in ray florets. Bars $=1 \mathrm{~cm}$. 


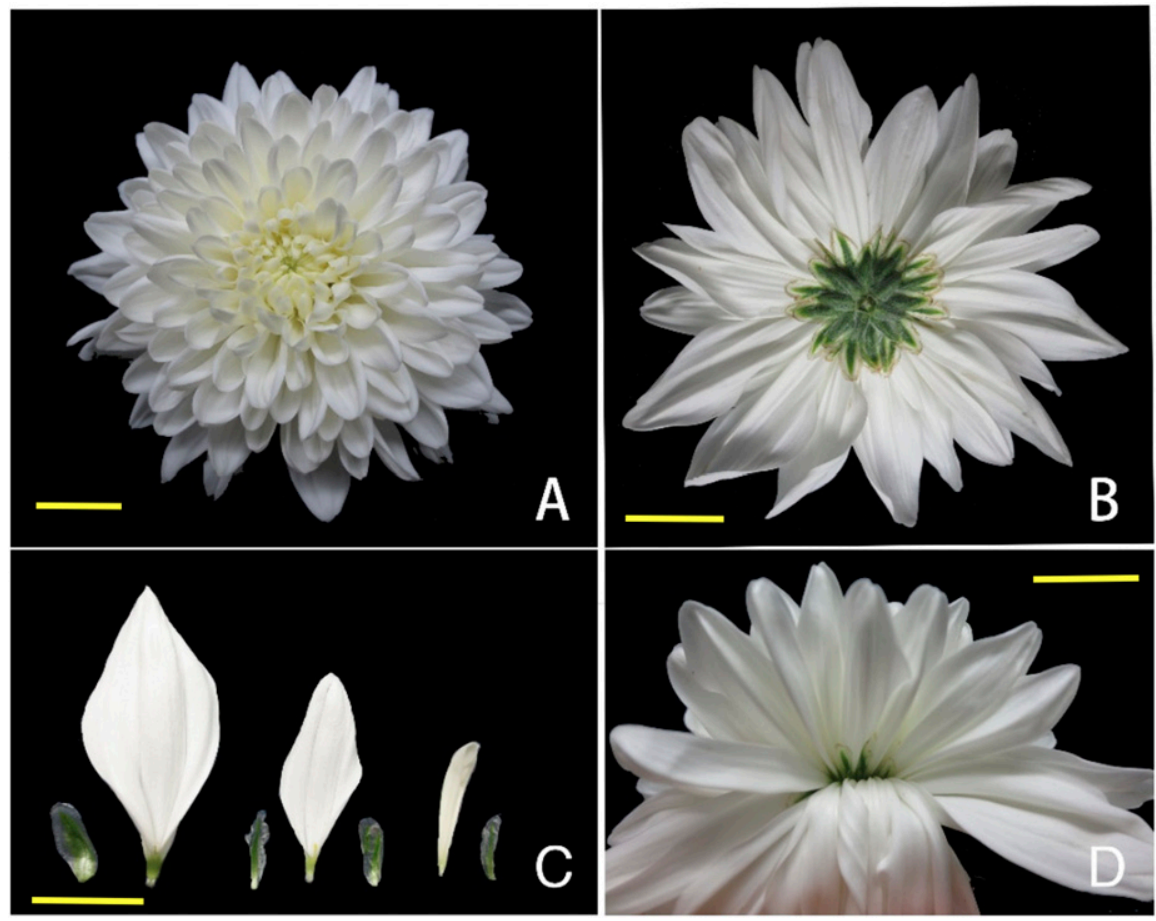

Fig. 10. Flower morphological variation in 'FGR-5'. (A) White capitulum. (B) Total bracts on the ray flowers. (C) Different size bracts. (D) Bracts between the ray flowers. Bars $=1 \mathrm{~cm}$.

we observed the early flower phenotype, flowering induction and flower development had been completed. Because of this process, genes that are regulated during the flowering induction process are more difficult to capture.

It is difficult to screen for candidate genes regulated by DNA methylation using early flower mutants. If the effects of a DNA methylation reduction on plant flowering are to be stabilized, there must be an apparent mutant strain (i.e., the line that displays early flowering after multiple passages). However, 5-azaC affects nongenetic DNA methylation, especially in genes that are transiently demethylated during flowering induction. It is difficult to capture this result in the offspring because the trait can be inherited, and this study cannot be further developed.

\section{Detection of methylation levels and their necessity}

In previous studies, scholars generally used methylation-sensitive amplified polymorphisms and liquid chromatography to detect methylation levels ( $\mathrm{Li}$ et al., 2017; Wang et al., 2009). The classic method is the detection of DNA methylation levels in an entire genome. It has been reported that the overall methylation levels in a plant during the flowering process is reduced; in fact, methylation and demethylation occur in the same time. The specific role of individual genes remains unknown. In the early flowering lines obtained after the early flower phenotype was observed, the flowering induction process was complete and the flower development process began. The methylation levels of the genes that changed during flowering will return to preflowering levels after flowering. Changes in the methylation levels of key genes should be examined. However, it is not clear which genes are regulated by DNA methylation in the process of flowering induction, and the next stage of work is difficult to perform. Therefore, screening flowering-inducing genes that may be regulated by DNA methylation should be performed. Finally, we should consider the use of stable floral lines with early flowering to examine candidate genes in the flowering process based on the screening candidate genes. The detection of the methylation status determines the specific mechanism of the early flower phenotype exhibited by the early flower line.

\section{Literature Cited}

Blümel, M., N. Dally, and C. Jung. 2014. Flowering time regulation in crops-what did we learn from Arabidopsis? Curr. Opin. Biotechnol. 32C:121-129.

Bratzel, F. and F. Turck. 2015. Molecular memories in the regulation of seasonal flowering: From competence to cessation. Genome Biol. 16:14.

Burn, J.E., D.J. Bagnall, J.D. Metzger, E.S. Dennis, and W.J. Peacock. 1993. DNA methylation, vernalization, and the initiation of flowering. Proc. Natl. Acad. Sci. USA 90(1):287-291.

Chen, F. and Z.C. Wang. 2011. Effects of 5-azaC on development and DNA methylation in wheat. J Henan University, Nat. Sci. 41(1): 61-66 (in Chinese with an English abstract).

Crevillén, P., H.C. Yang, X. Cui, C. Greeff, M. Trick, Q. Qi, X.F. Cao, and C. Dean. 2014 Epigenetic reprogramming that prevents transgenerational inheritance of the vernalized state. Nature 515(7528):587-590
Cubas, P., C. Vincent, and E. Coen. 1999. An epigenetic mutation responsible for natural variation in floral symmetry. Nature 401(6749): 157-161.

Deng, W.W. and X.F. Cao. 2007. Involvement of the histone acetyltransferase AtHACl in the regulation of flowering time via repression of FLOWERING LOCUS C in Arabidopsis. Plant Physiol. 143(4):1660-1668.

Fieldes, M.A. and L.M. Amyot. 1999. Epigenetic control of early flowering in flax lines induced by 5 -azacytidine applied to germinating seed. $\mathrm{J}$. Hered. 90(1):199-206.

Fraga, H.P.F., L.N. Vieira, C.A. Caprestano, D.A Steinmacher, G.A. Micke, D.A. Spudeit, R. Pescador, and M.P. Guerra. 2012. 5-azacytidine combined with 2,4-d improves somatic embryogenesis of Acca sellowiana, (O. Berg) Burret by means of changes in global DNA methylation levels. Plant Cell Rep. 31(12):2165-2176.

Gao, J.Y. 2012. The effects of genome-wide DNA methylation on vernalization performance in Brassica napus L. [master's thesis]. Zheng Zhou, Zheng Zhou University (in Chinese).

He, Y.K. and J.Y. Li. 2001. Differential expression of triplicate phosphoribosylanthranilate isomerase isogenes in the tryptophan biosynthetic pathway of Arabidopsis thaliana(1.)Heynh. Planta 212(5-6):641-647.

Jacobsen, S.E., H. Sakai, E.J. Finnegan, X.F. Cao, and E.M. Meyerowitz. 2000. Ectopic hypermethylation of flower-specific genes in Arabidopsis. Curr. Biol. 10(4):179-186.

Jacobsen, S.E. and E.M. Meyerowitz. 1997. Hypermethylated superman epigenetic alleles in Arabidopsis. Science 277(5329):1100-1103.

Kim, D.H. and S. Sung. 2014. Genetic and epigenetic mechanisms underlying vernalization. Arabidopsis Book 12(6016):E0171.

Kondo, H., H. Ozaki, K. Itoh, A. Kato, and K. Takeno. 2006. Flowering induced by 5-azacytidine, a DNA demethylating reagent in a short-day plant, Perilla frutescens var. crispa. Physiol. Plant. 127(1):130-137.

Kondo, H., T. Miura, K.C. Wada, and K. Takeno. 2007. Induction of flowering by 5-azacytidine in some plant species: Relationship between the stability of photoperiodically induced flowering and flower-inducing effect of DNA demethylation. Physiol. Plant. 131(3):462-469.

Kumpatla, S.P. and T.C. Hall. 1998. Longevity of 5 -azacytidine-mediated gene expression and re-establishment of silencing in transgenic rice. Plant Mol. Biol. 38(6):1113-1122.

Li, N., Y. Zhang, L.N. Xie, and Y.H. Li. 2012 Research progress in DNA methylation in plants. Plant Physiology J. 11:1027-1036 (in Chinese with an English abstract).

Li, S.F., G.J. Zhang, J.H. Yuan, C.L. Deng, L.D. Lu, and W.J. Gao. 2015. Effect of 5-azaC on the growth, flowering time and sexual phenotype of spinach. Russ. J. Plant Physiol. 62(5):670 675.

Li, S.L., M.M. Li, Z.A. Li, Y. Zhu, H.X. Ding, X.X. Fan, F. Li, and Z.C. Wang. 2019. Effects of the silencing of CmmET1 by RNA interference in chrysanthemum (Chrysanthemum morifolium). Plant Biotechnol. Rep. 13(1):63-72.

Li, Z.A., J. Li, Q.Q. Zhu, Y.H. Liu, and Z.C. Wang 2017. Effect of exrernal 5-azaC on physiology and DNA methylation and gene-expression of Chrysanthemum. J Henan University, Nat. Sci. 47(2):162-169 (in Chinese with an English abstract).

Martienssen, R. 1996. Epigenetic phenomena: Paramutation and gene silencing in plants. Curr. Biol. 6(7):810-813. 
Song, Y.P. 2013. Genetic regulation of floral development in Populus tomentosa [Ph. D. Dissertation]. Beijing. Beijing Forestry University (in Chinese with an English abstract).

Sun, Z.C., J.Q. Huang, and Z.J. Wang. 2015. Research advance on microRNA of plant flower development. Mol. Plant Breed. 3(5): 1177-1184 (in Chinese with an English abstract).

Teotia, S. and G.L. Tang. 2015. To bloom or not to bloom: Role of microRNAs in plant flowering. Mol. Plant 8(3):359-377.

Tyunin, A.P., K.V. Kiselev, and Y.N. Zhuravlev. 2012. Effects of 5-azacytidine induced DNA demethylation on methyltransferase gene expression and resveratrol production in cell cultures of Vitis amurensis. Plant Cell Tissue Organ Cult. 111(1):91-100.

Tatra, G.S., J. Miranda, C.C. Chinnappa, and D.M. Reid. 2000. Effect of light quality and 5-azacytidine on genomic methylation and stem elongation in two ecotypes of Stellaria longipes. Physiol. Plant. 109(3): 313-321.

Wang, Z.C., L.J. Nie, and Y.X. He. 2009. The effect of 5-azactidine to the DNA methylation and morphogenesis character of Chrysanthemum during in vitro growth. Acta Horticulturae Sinica. 36(12):1783-1790 (in Chinese with an English abstract).

Xiao, Q.Q. 2010. Fine-mapping of qFT.A10 and dissection the relationship between DNA methylation of BnA10.FLC and flowering in Brassica Napus [master's] Wu Han. Huazhong Agricultural University (in Chinese with an English abstract).

Yuan, J.L., G.P. Guo, J.J. Yue, X.J. Wu, and X.P. Gu. 2012. Features of DNA methylation during the flowering process of Banbusa multiplex.
Acta Botanica Boreali-Occidentalia Sinica 01:60-66 (in Chinese with an English abstract).

Zhang, S.L. and S.L. Dai. 2013. Chinese Chrysanthemum Book. China Forestry Publishing House, Beijing (in Chinese).

Zhou, D.X. 2009. Regulatory mechanism of histone epigenetic modifications in plants. Epigenetics 4(1):4.

Zhu, Q.Q., Z.A. Li, Y.X. He, W.Q. Zhao, and Z.C. Wang. 2013. Research progress on epigenetic and flowering-time regulation. Acta Horticulturae Sinica. 40(9):001787-001794 (in Chinese with an English abstract).

Zhu, Q.Q. 2014. Effect of 5-azaC on DNA methylation and gene-expression of chrysanthemum. [Master's Thesis]. Henan University (in Chinese).

Zografou, T. and F. Turck. 2013. Epigenetic control of flowering time. Epigenetic Memory Control Plants. 18:77-105. 